\title{
Astragalus polysaccharides alleviates glucose toxicity and restores glucose homeostasis in diabetic states via activation of AMPK
}

\author{
Feng ZOU, Xian-qing MAO, Nian WANG, Jian LIU, Jing-ping OU-YANG* \\ Department of Pathophysiology, Medical College of Wuhan University, Wuhan 430071, China
}

\begin{abstract}
Aim: To establish the mechanism underlying the improvement of glucose toxicity by Astragalus polysaccharide (APS), which occurred via an AMP activated protein kinase (AMPK)-dependent pathway.

Methods: In vivo and in vitro effects of APS on glucose homeostasis were examined in a type 2 diabetes mellitus (T2DM) rat model. The T2DM rat model was duplicated by a high-fat diet (58\% fat, $25.6 \%$ carbohydrate, and $16.4 \%$ protein) and a small dose of streptozotocin (STZ, $25 \mathrm{mg} / \mathrm{kg}$, ip). After APS therapy $\left(700 \mathrm{mg} \cdot \mathrm{kg}^{-1} \cdot \mathrm{d}^{-1}\right.$, ig) for 8 weeks, blood glucose, glycosylated hemoglobin, and serum insulin were measured. Insulin sensitivity was evaluated by the comprehensive analysis of oral glucose tolerance tests (OGTT) and HOMA IR index. Hepatic glycogen was observed by the PAS staining method. The expression and activity of skeletal muscle AMPK $\alpha$ and acetyl-CoA carboxylase (ACC), and the phosphorylation of hepatic glycogen synthase (GS), the glycogen synthase (GS), were measured by Western blotting. Glucose uptake was measured with the 2-deoxy-[ $\left.{ }^{3} \mathrm{H}\right]$ - $D$-glucose method in $\mathrm{C} 2 \mathrm{C} 12$ cells.

Results: The hyperglycemia status, insulin sensitivity, glucose uptake, and activation level of AMPK in diabetic rats were improved in response to APS administration. APS could also alleviate glucose toxicity in cultured mouse cells by the activation of AMPK.

Conclusion: APS can alleviate glucose toxicity by increasing liver glycogen synthesis and skeletal muscle glucose translocation in the T2DM rat model, via activation of AMPK.
\end{abstract}

Keywords: Astragalus polysaccharides; T2DM; AMPK; glucose; toxicity

Acta Pharmacologica Sinica (2009) 30: 1607-1615; doi: 10.1038/aps.2009.168

\section{Introduction}

Diabetes mellitus is a prevalent chronic disease in modern society. After heart disease and cancer, it represents the third cause of death in terms of human diseases. Type 2 diabetes mellitus (T2DM) is the major form of diabetes mellitus and is associated with insulin resistance, obesity, hyperglycemia, hyperlipidemia, and chronic inflammation, among other symptoms. With the increase in the number of elderly, the prevalence of obesity, and increasing incidence of more blood vessel diseases such as atherosclerosis, the number of T2DM patients is expected to reach 300 million by the year $2025^{[1]}$. T2DM has become a tremendous global health problem in the 21st century, especially with its multiple complications involving heart, kidney, foot, and retina, etc. Therefore, the prevention and therapy of T2DM is very important and urgent. Typical drug treatments for T2DM include insulin sensitizers such as rosiglitazone and absorption of glucose inhibitors such as

\footnotetext{
* To whom correspondence should be addressed.

E-mail jpoy@163.com

Received 2009-04-05 Accepted 2009-10-22
}

metformin ${ }^{[2,3]}$. Although effective at reducing the process of T2DM, these drugs also cause adverse side effects in the body. In contrast, traditional Chinese medicines and their extractions have proven availability and effectiveness to treat T2DM and its complications.

Radix Astragali (the root of Astragalus membranaceus Huangqi) is a common traditional Chinese medicine that has a long history as a Chinese herbal. It is used in the treatment of diseases of the heart, liver, and kidney. In western herbal medicine, Radix Astragali can also be used in immune system disorders, viral infections, and even cancer ${ }^{[4-6]}$. Of interest to the present study is that Radix Astragali has been reported to have an antidiabetic effect, although its pharmacological properties and the mechanisms underlying its antidiabetic effects are not yet clear. Astragalus polysaccharide (APS) is the polysaccharide component of the ethanol extract of Astragalus roots and is the active component of Astragalus. In a previous study, we found that treatment with APS lowers blood sugar by decreasing the expression and activity of protein-tyrosine phosphatase 1B (PTP1B) in the skeletal muscles of T2DM rats $^{[7]}$. We also found that APS can improve insulin sensitivity, 
which is coupled with an enhanced adaptive capacity of the endoplasmic reticulum ${ }^{[8]}$.

Blood glucose measurement reflects the amount of sugar being transported in vivo in the bloodstream. One of the main outlets for blood glucose is via transit to the liver and muscle for conversion to glycogen and storage. Another is utilization by cells, which then release energy by oxygenolysis for general metabolism. The glucose transporter 4 (GLUT4) is the key signaling molecule that coordinates glucose conversion and metabolism with glucose transport. In response to a signal, GLUT4 is translocated from the cytoplasm to the plasma membrane, where it facilitates the entry of glucose into the cell and produces a marked cellular response $\mathrm{e}^{[9,10]}$. When pathogenic factors disturb the translocation of GLUT4, the cells cannot utilize glucose, which accumulates and causes the harmful condition known as "glucose toxicity" [11]. In the case of T2DM, enhancement of glycogen synthesis and GLUT4 translocation, as well as augmentation of glucose uptake, have been strategies taken to avoid the glucose toxicity associated with diabetes.

In addition to the classical insulin signal pathway, the translocation of GLUT4 to the plasma membrane is also regulated by an adenosine monophosphate-activated protein kinase (AMPK) signal pathway ${ }^{[12]}$. AMPK is a type of highly conserved serine/threonine protein activating enzyme and is found primarily in the heart, skeletal muscle, liver, pancreas, etc. AMPK belongs to a family of energy sensing enzymes that are activated under conditions signifying cellular stress. These stresses usually result in a change in the intracellular ATP-toAMP ratio, thus the AMPK pathway acts essentially like a "fuel gauge". Following activation, AMPK functions to restore cellular ATP by inhibiting ATP consumption as well as accelerating ATP generation. AMPK activity can also cause insulin sensitization and can modulate blood glucose levels through its action on liver and muscle ${ }^{[13]}$. AMPK activation also regulates lipid oxidation and synthesis.

Another major action of AMPK is to stimulate GLUT4 translocation in muscle. Activation of AMPK increases glucose uptake and causes translocation of GLUT4 from microvesicles to the plasma membrane. Metformin and berberine have been shown to stimulate AMPK activity in muscle and liver cells via discrete mechanisms ${ }^{[14,15]}$. This activation of AMPK can also increase the glycogen content and activity of glycogen synthase (GS), a key enzyme in glycogen synthesis ${ }^{[16,17]}$. The AMPK pathway is a potential target for therapeutic treatment of T2DM. Therefore, identification of novel AMPK activators would be a new strategy for the T2DM treatment.

The aim of this study was to determine whether APS plays a direct role in alleviating glucose toxicity in vivo and in vitro. In addition, we also investigated whether the hypoglycemic effects of APS involve the AMPK signal pathway.

\section{Materials and methods} Preparation of APS extracts

Astragalus membranaceus (Fisch) Bunge var mongholicus (Bunge) Hsiao were purchased from Shanghai Medicinal
Materials (Shanghai, China). Astragalus polysaccharide (APS) was extracted with optimized techniques using direct water decoction, as described previously ${ }^{[18,19]}$. The resulting polysaccharide extract was dialyzed against several changes of water and then lyophilized. Carbohydrate content of the final product was $96.1 \%$. Three subtypes of APS were defined by phytochemical screening: APS I, II, and III (1.47:1.21:1). APSI consisted of $D$-glucose, $D$-galactose, and L-arabinose in molar ratios of 1.75:1.63:1 and had an average molecular weight of $36300 \mathrm{kDa}$. Both APSII and APSIII were a-1,4-linked dextrans that contained exiguous a-1,6-linkages. APS was a hazelcolored and water-soluble powder. It was diluted to $12 \%$ in normal saline prior to use. The identity of the extract was confirmed by the Department of Authentication of Chinese Medicine, Hubei College of Chinese Traditional Medicine (Wuhan, China).

\section{Rat experiments}

Forty four male Sprague-Dawley (SD) rats (220-250 g) supplied by the Animal Experimental Center of Wuhan University (ABSL-3) were acclimatized in communal cages at $22^{\circ} \mathrm{C}$, with a 12-h light/12-h dark cycle, then were randomly divided into A group $(n=20)$ and B group $(n=24)$. Rats from A group were fed with standard chow diet, while rats from $B$ group were fed with a high-fat diet (58\% fat, $25.6 \%$ carbohydrate and $16.4 \%$ protein). At the end of the 8th week, rats from B group were treated with a small dose intraperitoneal injection of streptozocin $(\mathrm{STZ}, 25 \mathrm{mg} / \mathrm{kg})^{[20]}$. The OneTouch ${ }^{\oplus} \mathrm{Ultra}^{\circledR}$ Blood Glucose Test System Kit (Lifespan Company, USA) was used to detect blood glucose. We used the data of random blood glucose $(\geq 11.1 \mathrm{mmol} / \mathrm{L})$ and oral glucose tolerance tests (OGTT) as the standards for determination of successful induction of diabetes. The OGTT was performed after a $16 \mathrm{~h}$ overnight fast. Glucose $(2 \mathrm{~g} / \mathrm{kg})$ was administered orally and blood was collected from the orbital sinus at $0,30,60$, and $120 \mathrm{~min}$. The rats that were not diabetic were subsequently given a semi-dose intraperitoneal injection of STZ $(12.5 \mathrm{mg} / \mathrm{kg})$. Diabetic rats from the B group were randomly divided into two groups at the end of 9th week: diabetes mellitus group (DM group, $n=8$ ), diabetes mellitus+APS group (DA group, $n=8$ ). At the same time, the A group was randomly divided into another two groups: a control group (C group, $n=10)$, and a control+APS group (CA group, $n=10$ ). The DA and CA groups received

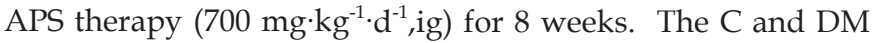
groups were given equal volumes of saline (Table 1). Body weight and blood glucose were recorded weekly. OGTT tests were performed after a $16 \mathrm{~h}$ overnight fast. Fasting blood glucose, serum insulin, and glycosylated hemoglobin $(\mathrm{GHb})$ were measured before euthanasia of the rats. Insulin content was determined by Radioimmunoassay (RIA). Insulin sensi-

Table 1. Time course of experimental animals.

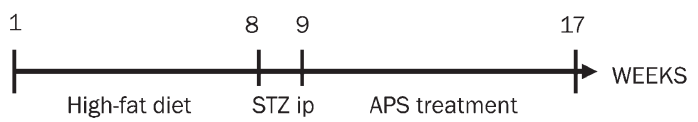


tivity was identified by the comprehensive analysis of OGTT and calculated values for HOMA-IR (HOMA-IR index=FPG (Fasting plasma glucose)[mmol/L]×FINS (fasting insulin) $[\mu \mathrm{U} / \mathrm{mL}] / 22.5)^{[21]}$. The rats were killed, then liver and skeletal muscle samples were rapidly excised and snap frozen in liquid nitrogen.

\section{Hepatic glycogen content assay and glycogen staining}

Fresh liver samples were put into phosphate buffered saline (PBS) to remove the blood, then weighed after drying sufficiently with filter paper. Glycogen was measured with a glycogen assay kit following the manufacturer's instructions. For hepatic glycogen staining, samples were fixed in $4 \%$ paraformaldehyde for $24-48 \mathrm{~h}$, then embedded in paraffin, and sectioned into $6 \mathrm{~mm}$ slices. Hepatic glycogen was stained with the periodic acid/leucofuchsin method (PAS) ${ }^{[22]}$. Prunosus particles were observed in the cytoplasm following staining.

\section{Preparation of plasma membranes of skeletal muscle}

Purified plasma membranes were disrupted by sonication to release caveolae and then subjected to linear sucrose gradient ultracentrifugation ${ }^{[23,24]}$. The cells from skeletal muscle were lysed on ice in $10 \mathrm{~mL}$ lysis Buffer A $(0.32 \mathrm{~mol} / \mathrm{L}$ sucrose, $5 \mathrm{mmol} / \mathrm{L}$ Tris- $\mathrm{HCl}$ (pH 7.5), $120 \mathrm{mmol} / \mathrm{L} \mathrm{KCl}, 1 \mathrm{mmol} / \mathrm{L}$ EDTA, $0.2 \mathrm{mmol} / \mathrm{L}$ PMSF, $1 \mu \mathrm{g} / \mathrm{mL}$ Leupeptin, and $1 \mu \mathrm{g} / \mathrm{mL}$ Aprotinin). Cell debris and nuclei were removed by centrifugation at $1000 \times g$ for $10 \mathrm{~min}$. A plasma membrane-containing pellet was obtained by centrifugation of supernatant at $15000 \times g$ for $30 \mathrm{~min}$. The pellet was resuspended in Buffer B (20 mmol/L HEPES, 10\% glycerol, 2\% Triton X-100, $1 \mathrm{mmol} / \mathrm{L}$ EDTA, $1 \mathrm{mmol} / \mathrm{L}$ EDTA, $0.2 \mathrm{mmol} / \mathrm{L}$ PMSF, $1 \mu \mathrm{g} / \mathrm{mL}$ Leupeptin, $1 \mu \mathrm{g} / \mathrm{mL}$ Pepstatin A, $1 \mu \mathrm{g} / \mathrm{mL}$ Aprotinin). The lysates were solubilized for $2 \mathrm{~h}$ at $4{ }^{\circ} \mathrm{C}$ and then centrifuged for $30 \mathrm{~min}$ at $10000 \times \mathrm{g}$. The supernatant was used as the purified plasma membrane preparation.

\section{Cell culture and pretreatment with APS}

Mouse C2C12 cells, the target cell line, were cultured in Dulbecco's modified Eagle's medium (DMEM) containing $10 \%$ fetal bovine serum supplemented with $100 \mathrm{U} / \mathrm{mL}$ penicillin, $100 \mu \mathrm{g} / \mathrm{mL}$ streptomycin, and $2 \mathrm{mmol} / \mathrm{L} \mathrm{L-glu-}$ tamine in a humidified atmosphere with $5 \% \mathrm{CO}_{2}$ at $37^{\circ} \mathrm{C}$. Differentiation into myotubes was induced as previously described $^{[25]}$. The cells were plated at a density of $3 \times 10^{4}$ cells $/ \mathrm{cm}^{2}$ and maintained in culture medium for $24 \mathrm{~h}$ before treatment. The medium was changed every $48 \mathrm{~h}$. The C2C12 cells were pretreated with APS (50-400 $\mu \mathrm{g} / \mathrm{mL})$ for $24 \mathrm{~h}$.

\section{MTT viability assays.}

The cell viability assay was based on the MTT (3-[4,5-2-yl]-2, 5-diphenyltetrazolium bromide) method. Briefly, $1 \times 10^{4} / \mathrm{mL}$ cells were cultured in each well of a 96-well microtiter plate and allowed to attach for $24 \mathrm{~h}$. The cells were then cultured in serum-free medium for 12-24 h and treated with various doses of APS (50-400 $\mu \mathrm{g} / \mathrm{mL}$ ) for another $24 \mathrm{~h}$. The media were removed and $20 \mu \mathrm{L}$ of $5 \mathrm{mg} / \mathrm{mL}$ MTT in PBS was added to each well, followed by incubation at $37^{\circ} \mathrm{C}$. After $4 \mathrm{~h}, 150 \mu \mathrm{L}$ of DMSO was added to dissolve the formazan crystals. The optical density was read at $570 \mathrm{~nm}$.

\section{Glucose uptake assay}

Glucose uptake in C2C12 cells was measured using the 2-deoxy- $\left[{ }^{3} \mathrm{H}\right]$-D-glucose method, as previously described ${ }^{[26]}$. The cells were cultured on 12-well cluster dishes, washed in phosphate-buffered saline (PBS) and incubated for $20 \mathrm{~min}$. The cells were then incubated for $20 \mathrm{~min}$ in PBS containing 50 $\mu \mathrm{mol} / \mathrm{L} 2$-deoxy-[ $\left.{ }^{3} \mathrm{H}\right]$ - $D$-glucose $(0.01 \mu \mathrm{Ci} / \mathrm{nmol})$. The reaction was terminated by placing the plates on ice and adding ice-cold PBS. After washing three times with PBS, the cells were dissolved in $0.1 \%$ SDS. The amounts of labeled glucose taken up by the cells were assessed by liquid scintillation counting.

\section{Western blotting analysis}

The cell lysates from liver, skeletal muscle or $\mathrm{C} 2 \mathrm{C} 12$ cells were prepared in $1 \mathrm{~mL}$ lysis buffer $(20 \mathrm{mmol} / \mathrm{L}$ Tris, $\mathrm{pH} 7.5$, $5 \mathrm{mmol} / \mathrm{L}$ EDTA, $10 \mathrm{mmol} / \mathrm{L} \mathrm{Na}_{4} \mathrm{P}_{2} \mathrm{O}_{7}, 100 \mathrm{mmol} / \mathrm{L} \mathrm{NaF}, 2$ $\mathrm{mmol} / \mathrm{L} \mathrm{Na}_{3} \mathrm{VO}_{4}, 1 \%$ Nonidet P-40, $1 \mathrm{mmol} / \mathrm{L}$ phenylmethylsulfonylfluoride (PMSF), and $10 \mu \mathrm{g} / \mathrm{mL}$ aprotinin) on ice in 1.5 $\mathrm{mL}$ microtubes. The lysates were solubilized by continuous stirring for $1 \mathrm{~h}$ at $4{ }^{\circ} \mathrm{C}$, then centrifuged for $10 \mathrm{~min}$ (cells) or $30 \mathrm{~min}$ (tissues) at $14000 \times \mathrm{g}$. The supernatants were collected and protein concentrations were measured with BCA protein assay reagent. The cell lysates were subjected to SDS-PAGE. After blotting, membranes were blocked with 5\% skim milk in Tris-buffered saline containing $0.1 \%$ Tween 20 for $1 \mathrm{~h}$ and then incubated with primary antibodies for $16 \mathrm{~h}$ at $4^{\circ} \mathrm{C}$. These included antibodies against AMPKa (Cell Signaling Technology), phosphor-AMPKa(Thr172) (Upstate), phosphor-acetylCoA carboxylase (Ser79) (Upstate), acetyl-CoA carboxylase (Upstate), GLUT4 (Cell Signaling Technology), phospho-glycogen synthase (Ser641) (Cell Signaling Technology), glycogen synthase (Cell Signaling Technology) and actin (Santa Cruz). After three washes in Tris-buffered saline containing $0.1 \%$ Tween 20, the membranes were incubated with anti-mouse or anti-rabbit IgG antibodies for $1 \mathrm{~h}$. The proteins were detected with an ECL system (KPL Amersham).

\section{Statistical analysis}

All values are expressed as mean \pm SEM. Statistical significance was determined using ANOVA followed by $t$-tests. $P<0.05$ was considered statistically significant.

\section{Results}

Effect of APS treatment on glucose metabolism and insulin sensitivity in a STZ-induced rat model

\section{Characteristics of the animal model}

To evaluate the in vivo effects of APS, we analyzed the basic indices of the animal models. Random blood glucose, fast blood glucose, glycosylated hemoglobin $(\mathrm{GHb})$ and OGTT were measured as routine protocols. At the end of the 9th week, we found that the random blood glucose levels of rats 
from the STZ-induced group (B group) were higher than those from the non-induced group (A group) $(22.13 \pm 3.11 \mathrm{mmol} / \mathrm{L}$ vs $6.1 \pm 0.52 \mathrm{mmol} / \mathrm{L}$ ). The OGTT readings at 30,60 , and $120 \mathrm{~min}$ from the STZ-induced group were all significantly higher than were those from the non-induced group (Figure 1). These results indicated that our animal model of T2DM was successfully established.

After treatment with APS $\left(700 \mathrm{mg} \cdot \mathrm{kg}^{-1} \cdot \mathrm{d}^{-1}, \mathrm{po}\right)$ for 8 weeks, there was a significant decrease in the levels of random and fasting blood glucose (Table 2, $P<0.05$ ). Moreover, $\mathrm{GHb}$ level from the DM group were higher than that in the $C$ group (Table 2, $P<0.05$ ), and the $\mathrm{GHb}$ of the DA group was lower than DM group (Table 2, $P<0.05$ ). Thus, APS had a good potency for mediating blood glucose levels and apparently could improve in vivo glucose homeostasis.

\section{Insulin sensitivity}

No significant differences in insulin levels were noted between the $C$ group and the DM group (Table $2, P>0.05$ ). In addition, treatment with APS had no effect on insulin levels in DM rats (Table 2, $P>0.05$ ). However, since the results of OGTT at all time points from the DA group were obviously lower than for the DM group (Figure $1, P<0.05$ ), we next calculated the HOMA-IR index among the four different groups. The HOMA-IR index of the DM group was higher than that in the $C$ group (Table 2, $P<0.05$ ). This supported the conclusion that our animal model was experiencing insulin resistance. The HOMA-IR index for the DA group was lower than for the DM group (Table 2, $P<0.05$ ).

With the results mentioned above, we concluded that APS had the potential of improving glucose homeostasis. In addition, APS could increase insulin sensitivity, but not through changing the insulin levels in the animal model.

\section{APS increases hepatic glycogen content and restores hepatic glucose homeostasis}

There was a significant reduction in hepatic glycogen content in the DM group (Figure $2 \mathrm{~A}, P<0.05$ ). After APS treatment, the numerical value in DA group increased (Figure 2A, $P<0.05)$. Consistent with this, hepatic glycogen staining also showed a significant augmentation of glycogen synthesis in DA group (Figure 2B). The hepatocytes of the DM group were clearly swollen and showed rarefaction of the cytoplasm. The number of glycogen particles also decreased in the DM group, as viewed under the microscope. Glycogen particles clearly
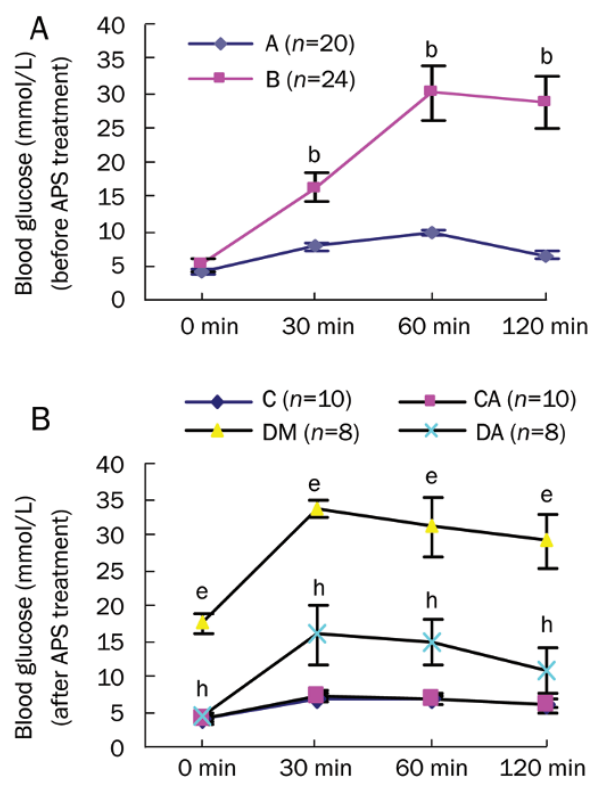

Figure 1. Oral glucose tolerance tests (OGTT) test of experimental animals at the end of 9th week (A) and at the end of 17th week (B). All data are expressed by Mean \pm SEM. ${ }^{b} P<0.05$ vs $A$ group. ${ }^{e} P<0.05$ vs $C$ group, ${ }^{\mathrm{h}} \mathrm{P}<0.05$ vs DM group (at the same age).

increased in the DA group (Figure 2B). Thus, APS treatment promoted glycogen synthesis in the livers of diabetic model rats.

When glycogen synthase is dephosphorylated at Ser641, the enzyme is activated ${ }^{[27]}$. APS treatment significantly reduced glycogen synthase phosphorylation at the Ser641 site (P-GS) (Figure 2C, $P<0.05$ ). The protein expression of GS showed no significant differences among the four groups (Figure 2C, $P>0.05)$.

\section{APS activates AMPK in skeletal muscle in T2DM rats}

AMPK is a key regulatory protein in glucose signal transduction. The protein expression of AMPKa showed no significant difference among the four groups (Figure 3, $P>0.05$ ). However, the phosphorylation of AMPKa (P-AMPKa) was significantly decreased in the skeletal muscle of the DM group compared to the $C$ group (Figure $3, P<0.05$ ). After APS treatment, the phosphorylation level was higher in the DA group than in the DM group (Figure 3, $P<0.05$ ).

Table 2. Characteristics of experimental animals at the end of 17 th week. All data are expressed by Mean \pm SEM. ${ }^{b} P<0.05$ vs $C$ group; ${ }^{e} P<0.05$ vs DM group (at the same age).

\begin{tabular}{|c|c|c|c|c|}
\hline Group & $C(n=10)$ & $\mathrm{CA}(n=10)$ & $\mathrm{DM}(n=8)$ & $\mathrm{DA}(n=8)$ \\
\hline Random blood glucose ( $\mathrm{mmol} / \mathrm{L})$ & $5.45 \pm 0.21$ & $5.85 \pm 0.67$ & $26.3 \pm 1.41^{b}$ & $15.1 \pm 4.67^{\mathrm{e}}$ \\
\hline Fasting blood glucose (mmol/L) & $3.9 \pm 0.32$ & $3.75 \pm 0.67$ & $19.63 \pm 0.8^{b}$ & $4.4 \pm 1.3^{\mathrm{e}}$ \\
\hline Blood serum insulin $(\mu \mathrm{U} / \mathrm{mL})$ & $13.26 \pm 0.25$ & $13.26 \pm 0.43$ & $12.41 \pm 1.83$ & $15.43 \pm 1.896$ \\
\hline $\mathrm{GHb}(\%)$ & $3.75 \pm 0.212$ & $3.75 \pm 0.071$ & $6.967 \pm 0.404^{b}$ & $5.3 \pm 0.762^{\mathrm{e}}$ \\
\hline HOMA-IR index & $2.04 \pm 0.13$ & $2.05 \pm 0.44$ & $6.32 \pm 0.86^{\mathrm{b}}$ & $5.14 \pm 0.90^{\mathrm{e}}$ \\
\hline
\end{tabular}


A

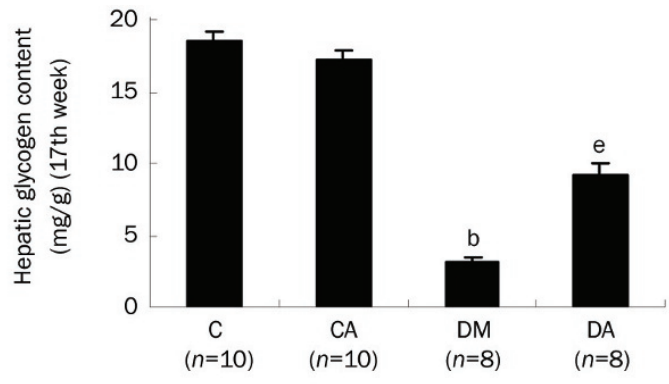

C
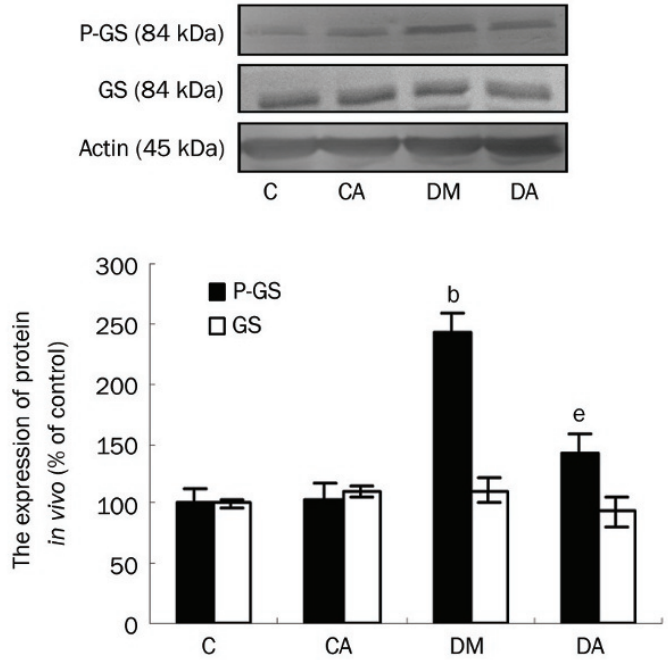

B
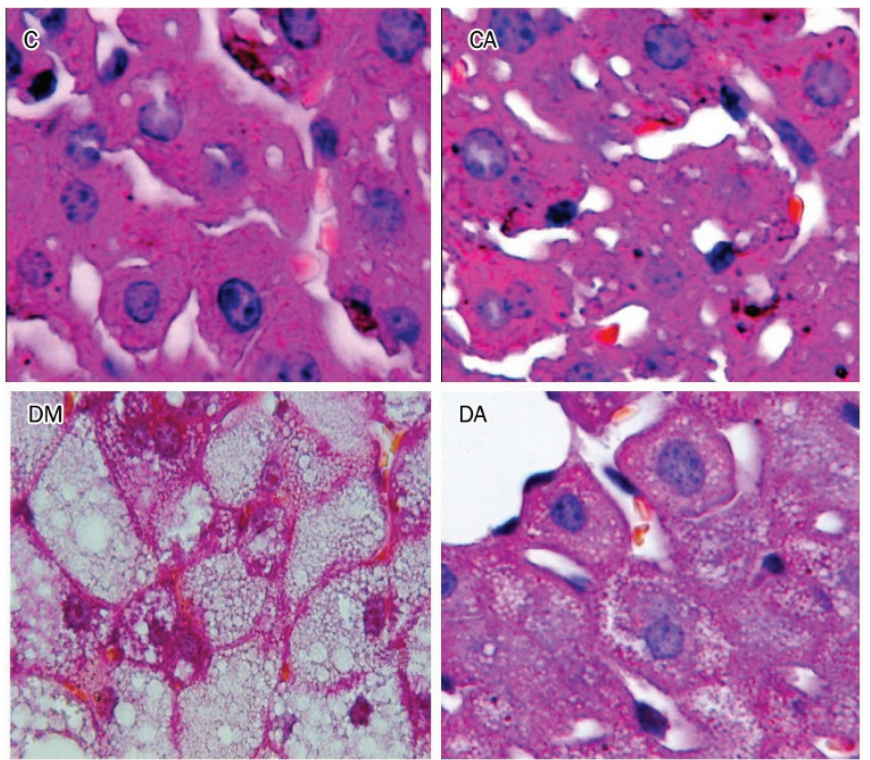

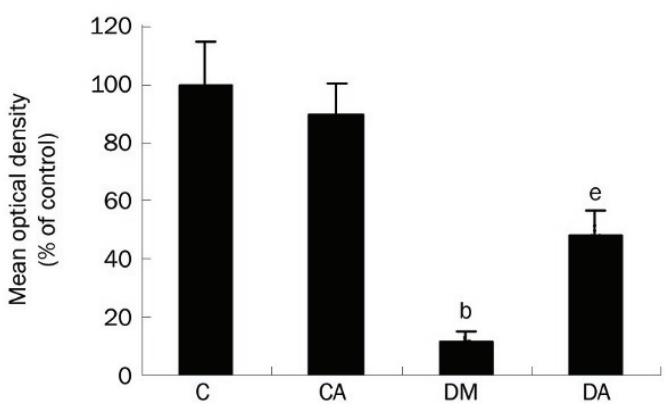

Figure 2. Effect of APS on the content of hepatic glycogen and expression of protein of glycogen synthase (GS) and P-GS in the liver of animal models by Western immunoblotting. (A) Effect of APS on the content of hepatic glycogen in the liver of animal models. (B) Effect of APS on the hepatic glycogen in the liver of animal models $(\times 1000, n=3)$. (C) Effect of APS on the expression of P-GS and GS in the liver of animal models ( $n=3)$. All data are expressed by mean \pm SEM. ${ }^{b} P<0.05$ vs $C$ group (at the same age). ${ }^{e} P<0.05$ vs $\mathrm{DM}$ group (at the same age).

Acetyl-CoA carboxylase (ACC) is the direct substrate of AMPK. The phosphorylation of ACC indicated the activation of AMPK. The expression of phosphorylated ACC (P-ACC) was lower in the DM group than in the $C$ group (Figure 3, $P<0.05)$, but still higher in the DA group than in the DM group (Figure 3, $P<0.05$ ). The protein expression of ACC showed no significant differences among the four groups (Figure 3, $P>0.05)$.

\section{APS increases the expression of GLUT4 of plasma membrane in skeletal muscle in T2DM rats}

The expression of GLUT4 in plasma membrane extracts and cell extracts of the DM group was lower than in the C or CA group (Figure $4, P<0.05$ ). The expression in the DA group was notably higher than in the DM group (Figure $4, P<0.05$ ). APS treatment increased the expression of GLUT4 in the plasma membrane extract and the cell extract, indicating stimulation of GLUT4 translocation in muscle.

Thus, APS treatment apparently increased the activity of AMPK and restored glucose homeostasis in liver and skeletal muscle in the animal model.
Effects of APS treatment on the C2C12 cell model and the high glucose-treated $\mathrm{C} 2 \mathrm{C} 12$ cell model

\section{APS activates AMPK in the C2C12 cell model}

When different concentrations of APS $(50,100,150,200$, or $400 \mu \mathrm{g} / \mathrm{mL}$ ) were provided to C2C12 cells, no toxicity was observed with the MTT assay for concentrations up to 200 $\mu \mathrm{g} / \mathrm{mL}$ (data not shown). Thus, concentrations of up to 200 $\mu \mathrm{g} / \mathrm{mL}$ were used in subsequent experiments. For dosedependency studies, cells were incubated in serum-free media for $8 \mathrm{~h}$, and then with the desired concentrations of APS for $48 \mathrm{~h}$. The activity and expression of AMPKa were measured by Western blotting of phosphorylated AMPKa (P-AMPKa) and AMPKa. A positive control, 5-amino-4imidazolecarboxamide ribonucleotide (AICAR), which can directly stimulate AMPK activity, was also included ${ }^{[28]}$. The activity of AMPK was increased by an increased concentration of APS (50-200 $\mathrm{\mu g} / \mathrm{mL}$ ) (Figure 5). No significant difference was observed in the expression of AMPKa among the four groups (Figure 5). 

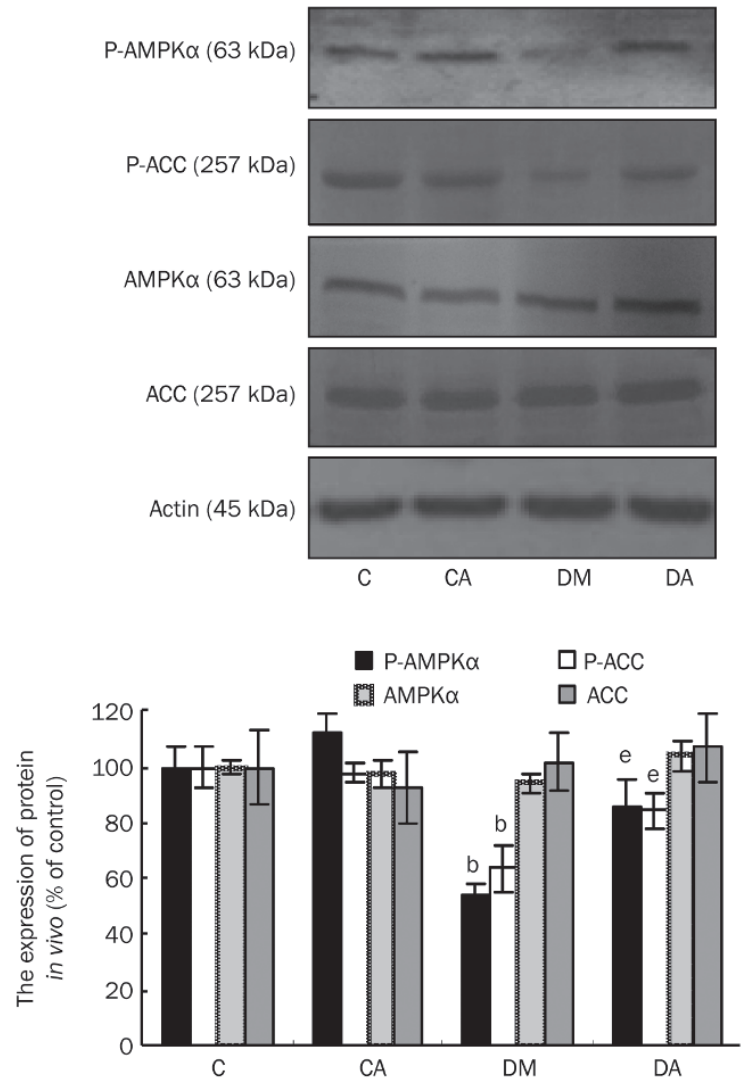

Figure 3. Effect of APS on the expression of protein of P-AMPK $\alpha, P-A C C$ AMPK $\alpha$ and Acetyl-CoA carboxylase (ACC) in the skeleton muscle of animal models by Western immunoblotting. All data are expressed by mean \pm SEM. $n=3$. ${ }^{\mathrm{b}} P<0.05$ vs $\mathrm{C}$ group (at the same age). ${ }^{\mathrm{e}} \mathrm{P}<0.05$ vs $\mathrm{DM}$ group (at the same age).
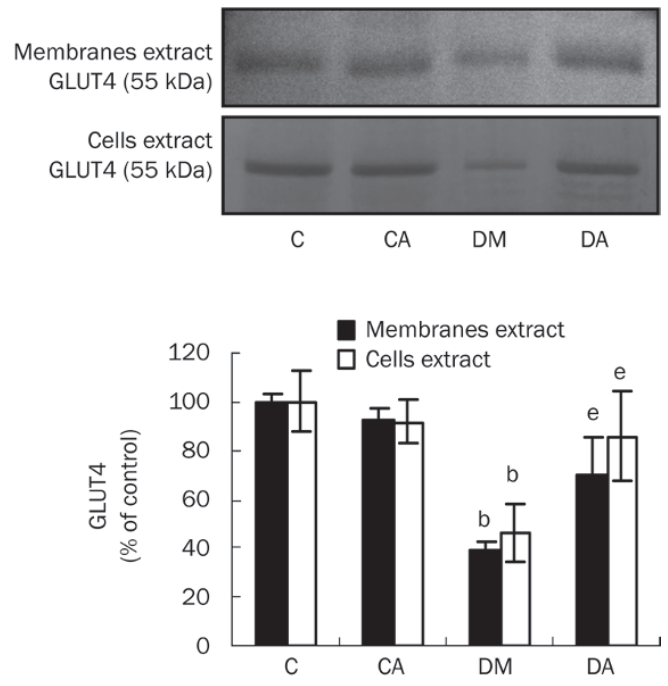

Figure 4. Effect of APS on the expression of protein of GLUT4 in the plasmalemma lysate of skeleton muscle extract and cells extract of animal models by Western immunoblotting. All data are expressed by mean \pm SEM. $n=3$. ${ }^{b} P<0.05$ vs $C$ group(at the same age); ${ }^{e} P<0.05$ vs DM group (at the same age).
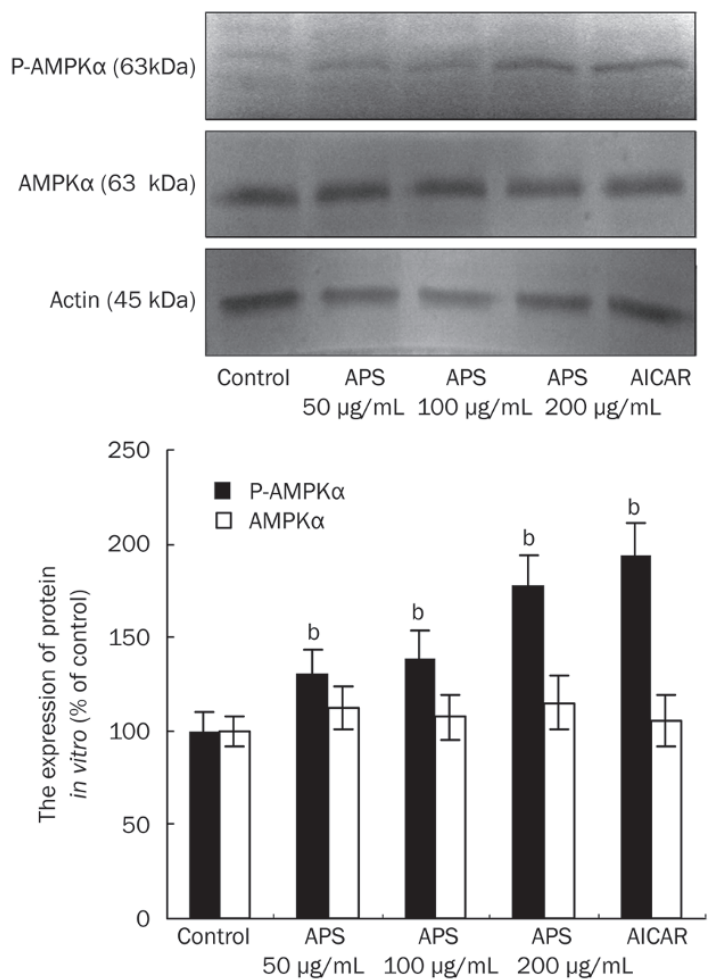

Figure 5. Effect of APS on the expression of protein of P-AMPK $\alpha$ and AMPK $\alpha$ in the APS-treated $\mathrm{C} 2 \mathrm{C} 12$ cell model by Western immunoblotting. Control cells were treated with the vehicle (DMSO) in an identical manner. Cells from APS group were treated with APS for $48 \mathrm{~h}$, cells from AICAR group were ere treated with AICAR $(0.5 \mathrm{mmol} / \mathrm{L})$ for $30 \mathrm{~min}$. All data are expressed by mean \pm SEM. $n=3$. ${ }^{b} P<0.05$ vs Control group.

\section{APS increased glucose uptake via the AMPK pathway}

APS at $200 \mu \mathrm{g} / \mathrm{mL}$ appeared to be the best concentration for showing significant effects on the activation of AMPK and this was used for all subsequent experiments. APS treatment increased glucose uptake significantly (Figure 6, $P<0.05$ ). We further attempted to determine whether APS-enhanced glucose uptake was mediated by AMPK. Compound C, which is a highly-selective AMPK inhibitor, as assessed by a direct enzyme assay ${ }^{[29]}$, had no effect on insulin-induced glucose uptake. It could, however, effectively block APS-induced glucose uptake (Figure 6, $P<0.05$ ), indicating involvement of the AMPK pathway in these APS effects.

\section{Effect of APS treatment on the high glucose-treated C2C12 cell} model

High glucose treatment (HG, $30 \mathrm{mmol} / \mathrm{L}$ ) of $\mathrm{C} 2 \mathrm{C} 12$ cells induced a reduction in AMPK activity. Treatment with APS increased AMPK activity in the HG cell group (Figure 7A). APS also increased glucose uptake into the HG cell group. However, this effect could be suppressed by the AMPK inhibitor, Compound C (Figure 7B).

In a conclusion, APS could alleviate glucose toxicity through increases in the AMPK pathway activity in the $\mathrm{C} 2 \mathrm{C} 12$ cell model. 


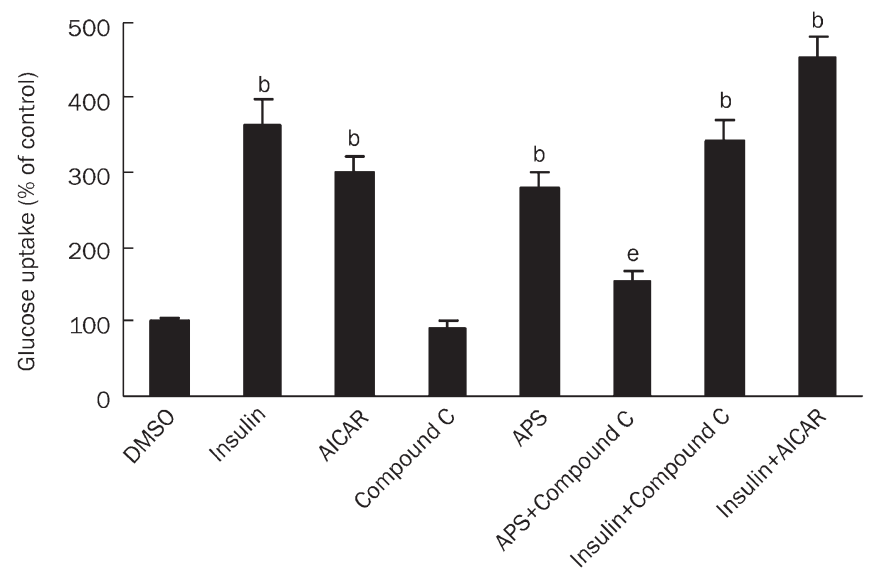

Figure 6. Concentration for insulin (100 nmol/L, $15 \mathrm{~min})$, APS (200 $\mu \mathrm{g} /$ $\mathrm{mL}, 48 \mathrm{~h}$ ), AICAR (5 mmol/L, $30 \mathrm{~min})$, and Compound C (20 $\mu \mathrm{mol} / \mathrm{L}, 30$ min) treatment on 2-deoxy- $\left[{ }^{3} \mathrm{H}\right]$-D-glucose uptake in differentiated $\mathrm{C} 2 \mathrm{C} 12$ cells. All data are expressed by mean \pm SEM. $n=4$. ${ }^{\mathrm{b}} P<0.05$ vs DMSO group; ${ }^{e} P<0.05$ vs APS group.

\section{Discussion}

T2DM has already become one of the most prevalent metabolic diseases in the world. The global extent of diabetes in adults is expected to increase from 5 to $6.2 \%$ between 2003 and 2025. Of more concern, children and adolescents have now also begun to be affected, due in large part to childhood obesity that is currently increasing in many countries ${ }^{[30,31]}$. Insulin resistance is the crucial link in T2DM. This resistance can be directly induced by hyperglycemia, and it is often associated with an imbalance between pancreatic endocrine function and the insulin sensitivity of insulin responsive tissues. Glucose toxicity, which is caused by hyperglycemia, has become an important cornerstone in the development of T2DM. Among the three major insulin responsive tissues (fat, muscle, and liver), the liver and muscle play the central role in the control of glucose homeostasis, balancing glycogen synthesis in liver and glucose transport in muscle. As a result, controlling these functions may efficiently resolve glucose toxicity.

In the present study, we adopted a small dose intraperitoneal injection of STZ (25 mg/ kg) and a high-fat diet after 8 weeks to duplicate the animal model of T2DM. The small dose injection of STZ can selectively impair pancreatic $\beta$ cells. Coupled with a prolonged period of a high-fat diet, the rats in the present study rapidly developed T2DM. The successful diabetic animal models showed hyperglycemia, high postprandial blood glucose, and high glycosylated hemoglobin, but had normal levels of insulin compared with normal rats. We considered that the animals were in a stage of insulin resistance that reflected relative insulin deficiency.

Astragalus membranaceus has been previously shown to have beneficial effects on antioxidant, antihypertensive, and immunomodulatory functions. The primary source of APS is the root of this herb, which is also the plant part that has benefits in traditional medicine. Consistent with previous studies, we provided evidence that APS has significant blood glucose
A
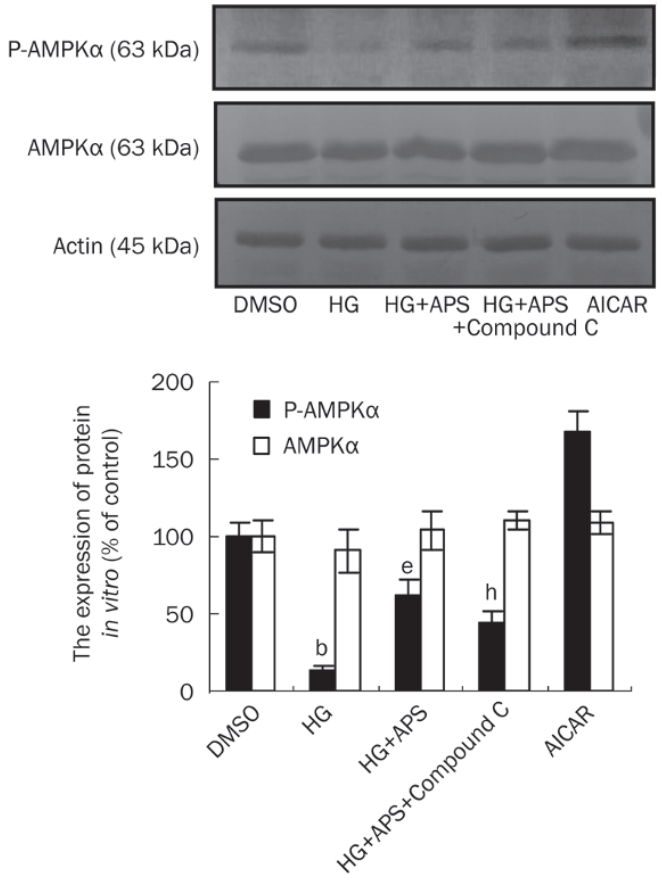

B

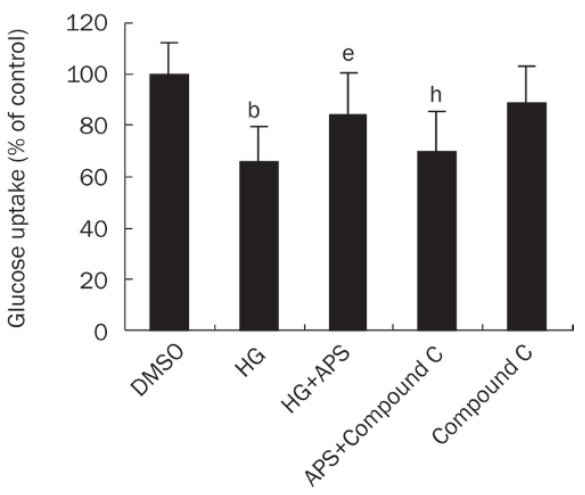

Figure 7. Effect of APS on the high glucose (HG)-treated $\mathrm{C} 2 \mathrm{C} 12$ cell model. Analysis of the expression of protein of P-AMPK $\alpha$ by Western immunoblotting and glucose uptake by 2-deoxy-[ $\left.{ }^{3} \mathrm{H}\right]-\mathrm{D}$-glucose method. (A) Effect of APS on the expression of P-AMPK $\alpha$ of high glucose-treated $\mathrm{C} 2 \mathrm{C} 12$ cell model $(n=3)$. (B) Effect of APS on the glucose uptake of high glucosetreated $\mathrm{C} 2 \mathrm{C} 12$ cell model $(n=4)$. All data are expressed by mean \pm SEM. ${ }^{\mathrm{b}} P<0.05$ vs DMSO group; ${ }^{\mathrm{e}} P<0.05$ vs HG group, ${ }^{\mathrm{h}} P<0.05$ vs HG+APS group.

lowering activity and insulin-sensitizing effects in our animal model. We use the value of random blood glucose to observe the condition of animals in general for APS treatment. It is not the "gold standard" because it has a fluctuation by the influence with the animals which just have diet. However, APS could decrease the random blood glucose significantly in contrast to diabetes group. APS could also improve the hepatic glycogen content and increase glycogen synthesis. Most importantly, we showed that APS strongly activates AMPK activity in both the in vivo animal model and the in vitro cell model.

Recently, AMPK has become the focus of a great deal of attention as a novel therapeutic target for the treatment of 
metabolic syndromes, especially T2DM ${ }^{[13]}$. AMPK has been demonstrated to mediate a number of pharmacological and physiological factors that exert beneficial effects on T2DM. Thus, the discovery of any novel activator of the AMPK pathway could contribute significantly to the prevention and treatment of T2DM. AMPK activation can cause insulin sensitization and modulate plasma glucose level through its action on liver and muscle, which can exert beneficial effects on type 2 diabetes patients ${ }^{[14,15]}$. Our present data indicate that AMPK is the major pathway affected by APS treatment for alleviating glucose toxicity. APS induced a variety of beneficial metabolic effects that were consistent with AMPK activation, including activation of phosphorylation of ACC and activation of GLUT4 translocation.

There are two major mechanisms for increasing glucose transport in skeletal muscle. One is the insulin signaling pathway, by which activation of PI-3 kinase/Akt phosphorylation leads to GLUT4 translocation to the plasma membrane ${ }^{[32]}$. The other is the AMPK signaling pathway. Increasing evidence suggests that AMPK mediates the effect of insulin-independent stimuli for glucose uptake $\mathrm{e}^{[33]}$. Indeed, the activation of AMPK in response to muscle contraction during hypoxia can be closely correlated with an increase in glucose uptake in the muscles. Initial work regarding the role of AMPK in the control of glucose uptake suggested that the activation of AMPK promotes the translocation of GLUT4 to the plasma membrane, via a PI-3 kinase-independent pathway.

Recently, several studies have suggested that AMPK may also enhance insulin sensitivity, both in vitro and in vivo. The interference of AICAR was shown to enhance insulinstimulated GLUT4 translocation and glucose uptake in Wistar rats $^{[34]}$. AMPK may also be activated via a PI-3 kinase pathway in vascular endothelial cells ${ }^{[35]}$. Some other reports suggest that AMPK functions upstream of Akt signaling ${ }^{[36]}$. A correlation does indeed exist between insulin and the AMPK signaling pathways. However, recent data on this subject are as yet highly controversial. In our study, we have demonstrated that APS induces glucose uptake in an AMPK-dependent pathway in $\mathrm{C} 2 \mathrm{C} 12$ cells. In a previous study, we found that APS can potentiate the insulin signal by activating Akt and therefore can increase the complexity of intercommunication between these two signals. However, these two signals, which are highly cell type-dependent and experimental conditiondependent, appear to have interaction with each other. As a result, further studies are required to elucidate the precise mechanism interconnecting these two signal pathways.

It is interesting to compare the pharmacological effects of APS with metformin, another significant insulin-sensitizing medicine. Based on the data presented in the current study, APS and metformin show a number of features in common. Metformin decreases blood glucose and improves insulin sensitivity in both human and animal models of insulin resistance. A major mode of action of metformin is via activation of AMPK ${ }^{[15]}$. APS treatment displays similar effects to those of metformin, but the mechanism by which APS activates AMPK is not yet known. Two different kinases, LKB1 and calmodu- lin-dependent protein kinase kinase (CAMKK) have recently been identified as novel upstream AMPK kinases ${ }^{[37,38]}$. LKB1 activates AMPK in response to energy stress and CAMKKb appears to be responsive to calcium changes, although their physiological relationship to the AMPK pathway will require further investigation ${ }^{[39,40]}$. In our future studies, we will investigate the mechanism of how APS actives AMPK, which may perhaps turn out to be via a similar mechanism to that of metformin.

In summary, our findings support the notion that the polysaccharides from Astragalus, a traditional Chinese herb, decrease blood glucose and improve insulin sensitivity in animal models of diabetic insulin resistance, at least in part by activating AMPK. Collectively, these effects lead to increased glycogen synthesis and glucose translocation, which can alleviate glucose toxicity in vivo and in vitro. APS clearly has potent antidiabetic effects and is a prime candidate for therapeutic trials for the treatment of T2DM.

\section{Acknowledgements}

We would like to thank all study sites and investigators who contributed to this study. This work was funded by grants from the National Natural Science Foundation of China (№ 30771023).

\section{Author contribution}

Jing-ping OU-YANG designed research; Feng ZOU, Jian LIU performed research; Xian-qing MAO, Nian WANG contributed new analytical tools and reagents; Feng ZOU, Xian-qing MAO, Nian WANG, Jing-ping OU-YANG analyzed data; Feng ZOU wrote the paper.

\section{References}

1 Kiberstis PA. A surfeit of suspects (special section-type 2 diabetes). Science 2005; 307: 1-2016.

2 Sewter C, Vidal-Puig A. PPARgamma and the thiazolidinediones: molecular basis for a treatment of 'Syndrome X'? Diabetes Obes Metab 2002; 4: 239-48.

3 Leverve XM, Guigas B, Detaille D, Batandier C, Koceir EA. Mitochondrial metabolism and type-2 diabetes: a specific target of metformin. Diabetes Metab 2003; 29: 6S88-94.

4 Grover JK, Yadav S, Vats V. Medicinal plants of India with antidiabetic potential. J Ethnopharmacol 2002; 81: 81-100.

5 Mao CP, Xie ML, Gu ZL. Effects of konjac extract on insulin sensitivity in high fat diet rats. Acta Pharmacol Sin 2002; 23: 855-9.

6 Wu F, Chen X. A review of pharmacological study on Astragalus membranaceus (Fisch) Bge. Zhong Yao Cai 2004; 27: 232-4.

7 Wu Y, Ou-Yang JP, Wu K, Wang Y, Zhou YF, Wen CY. Hypoglycemic effect of Astragalus polysaccharide and its effect on PTP1B. Acta Pharmacol Sin 2005; 26: 345-52.

8 Mao XQ, Wu Y, Wu K, OU-YANG JP. Astragalus polysaccharide reduces hepatic endoplasmic reticulum stress and restores glucose homeostasis in a diabetic KKAy mouse model. Acta Pharmacol Sin 2007; 28: $1947-56$.

9 Hudson AW, Ruiz ML, Birnbaum MJ. Isoform-specific subcellular targeting of glucose transporters in mouse fibroblasts. J Cell Biol 1992; 116: 785-97.

10 Haney PM, Slot JW, Piper RC, James DE. Intracellular targeting of the 
insulin-regulatable glucose transporter (glut4) is isoform specific and independent of cell type. J Cell Biol 1991; 114: 689-99.

11 Robertson RP, Harmon JS. Diabetes, glucose toxicity, and oxidative stress: a case of double jeopardy for the pancreatic islet $\beta$ cell. Free Radical Biol Med 2006; 41: 177-84.

12 Misra P, Chakrabarti R. The role of AMP kinase in diabetes. Indian J Med 2007; 125: 389-98.

13 Carling D. AMP-activated protein kinase: balancing the scales. Biochimie 2005; 87: 87-91.

14 Lee YS, Kim WS, Kim KH, Yoon MJ, Cho HJ, Shen Y, et al. Berberine, a natural plant product, activates AMP-activated protein kinase with beneficial metabolic effects in diabetic and insulin-resistant states. Diabetes 2006; 55: 2256-64.

15 Zhou G, Myers R, Li Y, Chen Y, Shen X, Fenyk-Melody J, et al. Role of AMP-activated protein kinase in mechanism of metformin action. J Clin Invest 2001; 108: 1167-74.

16 Bollen M, Keppens S, Stalmans W. Specific features of glycogen metabolism in the liver. Biochem J 1998; 336: 19-31.

17 Aschenbach WG, Hirshman MF, Fujii N, Sakamoto K, Howlett KF, Goodyear $\sqcup$. Effect of AICAR treatment on glycogen metabolism in skeletal muscle. Diabetes 2002; 5I: 567-73.

18 Shao BM, Xu W, Dai H, Gao XM. A study on the immune receptors for polysaccharides from the roots of Astralus membranaceus, a Chinese medicinal herb. Biochem Biophys Res Commun 2004; 320: 110311.

19 Zhang YW, Wu CY, Cheng JT. Merit of Astragalus polysaccharide in the improvement of early diabetic nephropathy with an effect on mRNA expressions of NF-KB and IKB in renal cortex of streptozotoxin-induced diabetic rats. J Ethnopharmacol 2007; 114: 387-92.

20 Srinivasan K, Viswanad B, Asrat L, Kaul CL, Ramarao P. Combination of high-fat diet-fed and low-dose streptozotocin-treated rat: a model for type 2 diabetes and pharmacological screening. Pharmacol Res 2005; 52: 313-20.

21 Bonora E, Targher G, Alberiche M. Homeostasis model assessment closely mirrors the glucose clamp technique in the assessment of insulin sensitivity studies in subjects with various degrees of glucose tolerance and insulin sensitivity. Diabetes Care 2000; 23: 57-63.

22 Ulusoy E, Eren B. Histological changes of liver glycogen storage in mice (Mus musculus) caused by high-protein diets. Histol Histopathol 2006; 21: 925-30.

23 Ortegren U, Yin L, Ost A, Karlsson H, Nystrom FH, Stralfors P. Separation and characterization of caveolae subclasses in the plasma membrane of primary adipocytes; segregation of specific proteins and functions. FEBS J 2006; 273: 3381-92.

24 Song KS, Li S, Okamoto T, Quilliam LA, Sargiacomo M, Lisanti MP. Co-purification and direct interaction of ras with caveolin, an integral membrane protein of caveolae microdomains. Detergentfree purification of caveolae membranes. J Biol Chem 1996; 271: 9690-7.

25 Epting CL, Lopez JE, Shen X, Liu L, Bristow J, Bernstein HS. Stem cell antigen-1 is necessary for cell-cycle withdrawal and myoblast differentiation in C2C12 cells. J Cell Sci 2004; 117: 6185-95

26 Tanishita T, Shimizu Y, Minokoshi Y, Shimazu T. The beta3-adrenergic agonist BRL37344 increases glucose transport into L6 myocytes through a mechanism different from that of insulin. J Biochem 1997; 122: 90-95.

27 Skurat AV, Roach PJ. Phosphorylation of sites 3a and 3b (Ser640 and Ser644) in the control of rabbit muscle glycogen synthase. J Biol Chem 1995; 270: 12491-7.

28 Sakoda H, Ogihara T, Anai M, Fujishiro M, Asano T. Activation of AMPK is essential for AICAR-induced glucose uptake by skeletal muscle but not adipocytes. Am J Physiol Endocrinol Metab 2002; 282: E123944.

29 Kim EK, Miller I, Aja S, Landree LE, Pinn M, McFadden J, et al. C75, a fatty acid synthase inhibitor, reduces food intake via hypothalamic AMP-activated protein kinase. J Biol Chem 2004; 279: 19970-6.

30 Rosenbloom AL, Joe JR, Young RS, Winter WE. Emerging epidemic of type 2 diabetes in youth. Diabetes Care 1999; 22: 345-54.

31 Schwartz MS, Chadha A. Type 2 diabetes mellitus in childhood: obesity and insulin resistance. J Am Osteopath Assoc 2008; 108: 518-24.

32 Wang L, Hayashi H, Ebina Y. Transient effect of platelet-derived growth factor on GLUT4 translocation in 3T3-L1 adipocytes. J Biol Chem. 1999; 274: 19246-53.

33 Russell RR 3rd, Bergeron R, Shulman GI, Young LH. Translocation of myocardial GLUT-4 and increased glucose uptake through activation of AMPK by AICAR. Am J Physiol 1999; 277: H643-9.

34 Yamaguchi S, Katahira H, Ozawa S, Nakamichi Y, Tanaka T. Activators of AMP-activated protein kinase enhance GLUT4 translocation and its glucose transport activity in 3T3-L1 adipocytes. Am J Physiol Endocrinol Metab 2005; 289: E643-9.

35 Zou MH, Kirkpatrick SS, Davis BJ, Nelson JS, Wiles WG 4th. Activation of the AMP-activated protein kinase by the anti-diabetic drug metformin in vivo. Role of mitochondrial reactive nitrogen species. J Biol Chem 2004; 279: 43940-51.

36 Ouchi N, Kobayashi H, Kihara S, Kumada M, Sato K, Inoue T. Adiponectin stimulates angiogenesis by promoting cross-talk between AMP-activated protein kinase and Akt signaling in endothelial cells. J Biol Chem 2004; 279: 1304-9.

37 Shaw RJ, Lamia KA, Vasquez D, Koo SH. The kinase lkb1 mediates glucose homeostasis in liver and therapeutic effects of metformin. Science 2005; 310: 1642-6.

38 Hawley SA, Pan DA, Mustard KJ, Ross L. Calmodulin-dependent protein kinase kinase-beta is an alternative upstream kinase for AMPactivated protein kinase. Cell Metab 2005; 2: 9-19.

39 Woods A, Dickerson K, Heath R, Hong SP. $\mathrm{Ca}^{2+} /$ calmodulin-dependent protein kinase kinase-beta acts upstream of AMP-activated protein kinase in mammalian cells. Cell Metab 2005; 2: 21-33.

40 Birnbaum MJ. Activating AMP-activated protein kinase without AMP. Mol Cell 2005; 19: 289-90. 